\title{
The Large-flowered Petunia (Petunia hybrida Vilm.) Genotype Promotes Expressions of Type-A Response Regulator and Cytokinin Receptor Genes like Cytokinin Response
}

\author{
Takaaki Nishijima ${ }^{1,2 *}$, Tomoya Niki ${ }^{1,2}$ and Tomoko Niki ${ }^{1}$ \\ ${ }^{I}$ National Institute of Floricultural Science, National Agriculture and Food Research Organization (NARO), Tsukuba 305-8519, Japan \\ ${ }^{2}$ Graduate School of Life and Environmental Sciences, University of Tsukuba, Tsukuba 305-8572, Japan
}

Expressions of the genes involved in the cytokinin (CK) phosphorelay signaling circuitry in petunia (Petunia hybrida Vilm.) were investigated with regard to the large-flowered trait induced by the semidominant Grandiflora $(G)$ gene. We isolated three cDNAs encoding type-A response regulators (RRs, PhRR1-3) and one cDNA encoding CK receptor histidine protein kinase $(P h H K)$. Expression levels of $P h R R 1-3$ and $P h H K$ in the developing corolla were markedly higher in large-flowered cultivars with the $G g$ genotype than in small- and medium-flowered cultivars with the $g g$ genotype. Higher expression levels of $P h R R 1-3$ and $P h H K$ in the $G g$ than in the gg genotypes was also consistent in $\mathrm{BC}_{4}$ plants in which the genetic background except for $G$ allele was mostly uniform. These results suggest that $G g$ genotype induces a high expression level of $P h R R 1-3$ and $P h H K$. These changes in expression caused by the $G$ allele were the same as those caused by the CK response. Since type-A RRs repress CK signaling, the $G$-allele-induced high expression levels of $P h R R 1-3$ presumably have partial inhibitory effects on $G$-alleleinduced corolla enlargement.

Key Words: feedback regulation, flower size, phosphorelay signaling circuitry, signal transduction.

\section{Introduction}

A large-flowered trait is an important breeding objective to improve the appearance of floricultural plants. This is clearly illustrated by the fact that many floricultural crops have much larger flowers than their corresponding wild species; however, large-flowered traits require a long time to develop, because of the rare occurrence of mutations responsible for large flowers (Nishijima, 2007).

Grandiflora $(G)$ is the only major gene inducing a large-flowered phenotype in petunia (Petunia hybrida Vilm.) (Ewart, 1984). Although the $G$ gene has not been molecularly identified, it is characterized as a semidominant gene, which mainly increases cell number rather than cell size to enlarge the corolla (Ewart, 1984; Nishijima et al., 2006). Commercial large-flowered cultivars are heterozygous, $G g$, because the homozygous

Received; January 27, 2011. Accepted; March 8, 2011.

This work was supported by the National Agriculture and Food Research Organization (Development of innovative crop through molecular analysis of useful genes) and JSPS KAKENHI (22580045).

* Corresponding author (E-mail: takaaki@affrc.go.jp).
$G G$ genotype has the pleiotropic effect of extremely low vigor (Ewart, 1984). Although $G G$ lines are necessary to produce commercial $\mathrm{F}_{1} G g$ seeds, the fertility of $G G$ plants is usually low.

Large flowers of petunia can also be induced by cytokinin (CK) application to the young flower buds (Nishijima et al., 2006). Expression of the gene encoding a CK biosynthesis enzyme, adenosine phosphateisopentenyltransferase (IPT), in the petunia corolla induces corolla enlargement through CK accumulation (Verdonk et al., 2008). In addition, the $G$ allele and CK induce other common morphological changes. Corolla enlargement induced by CK is attributed mainly to cell number increase as observed in corolla enlargement by the $G$ allele (Nishijima et al., 2006; Verdonk et al., 2008). The $G$ allele induces paler leaf and sepal colors, which is also a characteristic of $\mathrm{CK}$ application to leaves and sepals (Ewart, 1984; Nishijima et al., unpublished data); however, the physiological implications of this similarity have not been investigated.

We previously reported that large-flowered cultivars have lower concentrations of endogenous $\mathrm{CKs}$, including CK nucleobases, CK nucleosides, and CK glucosides, than small- and medium-flowered cultivars (Nishijima 
et al., 2011). Thus, the large-flowered phenotype induced by the $G$ allele is not attributable to high endogenous CK concentrations, although CK application effectively enlarges the corolla. The low CK concentration in largeflowered cultivars was caused by increased expression of the genes encoding CK oxidase/dehydrogenase (CKX) that degrade CKs through side-chain cleavage. This result enabled us to predict the involvement of CK signal transduction in $G$-allele-induced corolla enlargement because $C K X$ expression is promoted by the $\mathrm{CK}$ response (Kiba et al., 2005; Nishijima et al., 2011).

CK early signal transduction consists of a twocomponent circuitry (reviewed in Mizuno (2005) and Oka (2005)). CK receptors are histidine protein kinases (HKs) that phosphorylate histidine phosphotransfer proteins (HPs) on receipt of biologically active CK molecules. Phosphorylated HPs move into the nucleus and phosphorylate type-B response regulators (RR). Phosphorylated type-B RRs function as transcription factors regulating the expression of target genes. Phosphorylated type-B RRs also induce the expression of type-A RRs. Accumulated type-A RRs act as repressors of $\mathrm{CK}$ signaling mediating a negative feedback loop in the signaling circuitry.

Expression of Type-A RRs is rapidly and greatly induced by the CK response (Brandstatter and Kieber, 1998; Taniguchi et al., 1998). Expression of type-A RRs is also regulated in response to light through phytochrome B (Sweere et al., 2001), by the WUSCHEL transcription factor involved in meristematic competence (Leibfield et al., 2005) and by the AUXIN RESPONSE FACTOR5/MONOPTEROS (MP) transcription factor mediating auxin signalling (Zhao et al., 2010); however, the induction of type-A $R R$ expression caused by the CK response is very conspicuous. Expression of $A H K 4$, one of the genes encoding CK receptor HKs, is also conspicuously induced by the CK response (Kiba et al., 2004).

We investigated the effect of the petunia largeflowered $G g$ genotype on the regulation of CK phosphorelay signaling circuitry. Based on the results, the role of the CK phosphorelay signaling circuitry in $G$ alleleinduced corolla enlargement is discussed. Further, the potential modification of the CK phosphorelay signaling circuitry to produce the larger-flowered trait is discussed.

\section{Materials and Methods}

\section{Plant materials}

The cultivars listed in Table 1 were used in the experiment. Small- and medium-flowered cultivars belong to the multiflora group with the $g g$ genotype of the Grandiflora $(G)$ gene, while large-flowered cultivars belong to the grandiflora group with the $G g$ genotype (Ewart, 1984). Cultivars indicated by asterisks were used for detailed analysis. We also constructed backcross lines which mostly have an uniform genetic background except for $G$ allele. A large-flowered cultivar 'Fulcon Midblue' with $G g$ genotype was crossed with a doubled haploid line 'Mitchell' with $g g$ genotype. The $G g$ segregants were clearly distinguished from $g g$ segregants by pale-colored shoots, and thick and large sepals and petals. $G g$ plants were selected and backcrossed to 'Mitchell'. Appearance of $g g$ segregants at $\mathrm{BC}_{2}$ and further generations became undistinguishable from

Table 1. Petunia hybrida cultivars used in the experiment.

\begin{tabular}{llll}
\hline \hline \multicolumn{1}{c}{ Cultivar } & Type & Corolla size & \multicolumn{1}{c}{ Source } \\
\hline Fantasy Ivory*z & multiflora & small & Sakata Seed Co. \\
Fantasy Blue* & multiflora & small & Sakata Seed Co. \\
Baccara White* & multiflora & medium & Sakata Seed Co. \\
Baccara Blue Picote** & multiflora & medium & Sakata Seed Co. \\
Pearl White & multiflora & medium & Takii Seed Co. \\
Pearl Sky Blue & multiflora & medium & Sakata Seed Co. \\
Baccara Rose Morn & multiflora & medium & Sakata Seed Co. \\
Carpet Pink & multiflora & medium & M\&B Flora Co., Ltd. \\
Rondo Pink & multiflora & medium & Takii Seed Co. \\
Rondo Violet Imp & multiflora & medium & Takii Seed Co. \\
Fulcon White* & grandiflora & large & Sakata Seed Co. \\
Fulcon Midblue* & grandiflora & large & Sakata Seed Co. \\
Fulcon Pink Morn & grandiflora & large & Sakata Seed Co. \\
Carnival Red Star & grandiflora & large & Takii Seed Co. \\
Carnival Brush Pink & grandiflora & large & Takii Seed Co. \\
Supercascade Blue & grandiflora & large & M\&B Flora Co., Ltd. \\
Carnival Salmon & grandiflora & large & Takii Seed Co. \\
Dream White & grandiflora & large & M\&B Flora Co., Ltd. \\
\hline
\end{tabular}

\footnotetext{
${ }^{z}$ Cultivars indicated by asterisk were used for detailed analysis.

${ }^{y}$ Corolla diameters of small-, medium-, and large-flowered cultivars were $33.8-43.3 \mathrm{~mm}, 57.1-65.2 \mathrm{~mm}$, and 80.9$86.7 \mathrm{~mm}$ (average of 10 flowers per cultivar) under our growth conditions.
} 
'Mitchell'. $\mathrm{BC}_{4}$ plants with $g g$ and $G g$ genotypes were used for the experiment.

We planted single seedlings in plastic pots $(15 \mathrm{~cm}$ in diameter, $18 \mathrm{~cm}$ in height) filled with a horticultural medium (Kureha-Engei-Baido, Kureha Chemical Industry Co. Ltd., Tokyo, Japan). Plants were grown in an incubator set at $22.5^{\circ} \mathrm{C}$ with a $16-\mathrm{h}$ photoperiod provided by fluorescent lamps $\left(150 \mu \mathrm{mol} \cdot \mathrm{m}^{-2} \cdot \mathrm{s}^{-1} \mathrm{PPFD}\right)$.

Corollas at the following growth stages were used for the analysis of gene expression: stage 1, early cell proliferation stage; stage 2, late cell proliferation stage; stage 3, cell expansion stage; and stage 4, flower opening. The growth of the corolla is attributed mainly to cell proliferation in stages 1 and 2, while it is solely attributed to cell expansion in stage 3. Corolla lengths at stages 1 , 2 , and 3 were $4.5-5.5,7.5-8.5$, and $24-28 \mathrm{~mm}$, respectively. Excised corollas were immediately frozen in liquid nitrogen and stored at $-80^{\circ} \mathrm{C}$ until analysis. Stems, and young and mature leaves of $\mathrm{BC}_{4}$ plants were also used for analysis. Length of young leaves was 5$7 \mathrm{~mm}$, while that of mature leaves was more than $30 \mathrm{~mm}$.

\section{Molecular cloning of genes involved in CK phosphorelay signaling circuitry}

Total RNA was obtained from young corollas of petunia (P. hybrida cv. 'Baccara White' and 'Fulcon Midblue') using an RNeasy Plant Mini Kit (QIAGEN K. K., Tokyo, Japan). cDNA was synthesized using a 1st Strand cDNA Synthesis Kit for RT-PCR (AMV; Roche Applied Science, Indianapolis, USA). Degenerate primers corresponding to the amino acid sequences HVLAVDD (forward 5'-CAYGTIYTIGCIGTIGAYG AY-3') and (E/Q)AGEELC (reverse 5'-YTSIGCICCYTC YTCIARRCA-3') were used to isolate partial sequences of type-A $R R(P h R R)$ cDNAs. To isolate partial sequences of $\mathrm{CK}$ receptor $H K(P h H K)$ cDNAs, two sets of degenerate primers corresponding to the amino acid sequences T(A/S/T)FERPL (forward 5'-ACIDVITTYG ARMGICCIYT-3')/MPTRIEHS (reverse 5'-CATIGGIG TICKDATYTCRTG-3') for the first PCR and YAPVIF(A/S)Q (forward 5'-GARTAYGCICCIGTIATH TWYDS-3')/TALFQSKA (reverse 5'-GTIGCIARRAA YTGISWYTTIGC-3') for the second PCR were used as nested PCR. 5'- and 3'-RACE-PCR (Frohman et al., 1988) were performed using the CapFishing ${ }^{\mathrm{TM}}$ Fulllength cDNA Premix Kit (Seegene, Seoul, South Korea) based on the partial cDNA sequences. Full-length cDNA was obtained by PCR using the following primers: forward 5'-ACATACACATTACACACACTCCTCTCTT3' and reverse 5'-TGTGATTAAACAATATCATCGATG TGTT-3' for $P h R R 1$, forward 5'-ATAAGCACAACCCC ATGTTCTAAG-3' and reverse 5'-TCTAGGCCCCTCTT AGATTAGCAA-3' for PhRR2, and forward 5'-CTTTAC ACTTTCATTCTTGATTCTTCTT-3', reverse 5'-CAGG CATTGCTTGATCTACACAATA-3' for PhRR3, and forward 5'-AGAATAGTGAGAAGTGTTAGTAGTGAT GGG-3' and reverse 5'-TGGACCTCTCCTCCCCTTCT
RTAT-3' for PhHK. Phylogenic analysis of the deduced protein was conducted by the neighbor-joining method (Saitou and Nei, 1987).

Expression analysis of genes involved in CK phosphorelay signaling circuitry

To investigate PhRRs and PhHK expression levels in cultivars with different corolla sizes, cDNAs were obtained from corollas at stages $1-4$, as defined above. To investigate the effect of CK application on PhRRs and PhHK expressions, $30 \mu \mathrm{mol} \cdot \mathrm{L}^{-1} \mathrm{~N}$-benzylaminopurine (BA) was dissolved in $20 \%$ aqueous acetone containing $0.05 \%$ Triton X-100 (Wako Pure Chemical, Osaka, Japan). Corollas at stage 2 were treated with $4 \mu \mathrm{L} /$ corolla of BA solution. cDNAs were obtained from the corollas 0-48 $\mathrm{h}$ after BA application. For total RNA extraction for cDNA synthesis, we treated the samples with DNase to avoid potential contamination with genomic DNA.

Primers were designed to amplify $107-259 \mathrm{bp}$ fragments of the $3^{\prime}$ regions of the cDNAs. Primer sequences used for the quantitative real-time PCR reaction were as follows: forward 5'-CCCTCGCCATCA CTTGACCT-3' and reverse 5'-GATTGAGGGGGTTTG AGAACC-3' for PhRR1, forward 5'-AAGAAGAAGTA GCTGAGAAAATAGTCACAG-3' and reverse 5'-TCAT TAAGTCAAATAGCAGTAAGGCC-3' for PhRR2, forward 5'-GAGCACTCCACAAATGGCAA-3' and reverse 5'-CGGCATTGATTAGTTGACATCGT-3' for $P h R R 3$, and forward 5'-AAATGGCGTAAATGGCATAT GC-3' and reverse 5'-TGAGAGCAGCTGAATTGGTAG AATC-3' for $P h H K$. The primers used for expression analysis of the internal standard $P h U B Q$ were as described previously (Nishijima et al., 2011). Gene expression was quantified using a quantitative realtime PCR machine (LightCycler, Roche Diagnostics, Mannheim, Germany) and SYBR Premix Ex Taq (TakaraBio, Ohtsu, Japan). The reactions were cycled with a preincubation step of $10 \mathrm{~min}$ at $95^{\circ} \mathrm{C}$ followed by 50 cycles of $5 \mathrm{~s}$ at $95^{\circ} \mathrm{C}, 10 \mathrm{~s}$ at $55^{\circ} \mathrm{C}$, and $20 \mathrm{~s}$ at $72^{\circ} \mathrm{C}$, each. The raw data were analyzed with LightCycler software version 3.5 (Roche Diagnostics). The plasmids (pGEM-T Easy vector, Promega, Madison, USA) harboring cDNAs of PhRR1, PhRR2, PhRR3, PhHK, and $P h U B Q$ were used to obtain standard curves. The ratio of the expression of each gene to that of $P h U B Q$ was calculated. Expression analysis was conducted independently in triplicate.

\section{Results and Discussion}

Isolation of genes encoding type- $A R R$ and $C K$ receptor $H K$ and their response to $C K$

Three full-length cDNAs of type-A $R R$-like gene (PhRR1, AB610787; PhRR2, AB610788; PhRR3, $\mathrm{AB} 610789)$ and a $\mathrm{CK}$ receptor $H K$-like gene $(P h H K$, AB610790) were isolated from young corollas of petunia. The deduced PhRR proteins had a structure typical of type-A RR that consisted of a receiver domain 
and short C-terminal sequence (Brandstatter and Kieber, 1998; Taniguchi et al., 1998). The receiver domain had two critically conserved aspartates and a lysine (i.e., D32, D-86, and K-137 in PhRR1; D-17, D-80, and K-132 in PhRR2; D-40, D-94, and K-146 in PhRR3) (Imamura et al., 1999). The phylogenic relationship between PhRRs and Arabidopsis type-A RRs (ARRs) is shown in Figure 1A. PhRR1 belonged to the same clade as ARR3 and ARR4. PhRR2 belonged to the same clade as ARR8 and ARR9. The deduced amino acid sequence of PhRR3 was identical to that of ARR3, although the nucleotide sequence of $P h R R 3$ was substantially different from that of $A R R 3$.

The deduced PhHK proteins had a structure typical of $\mathrm{CK}$ receptor $\mathrm{HK}$ that was composed of the following

A

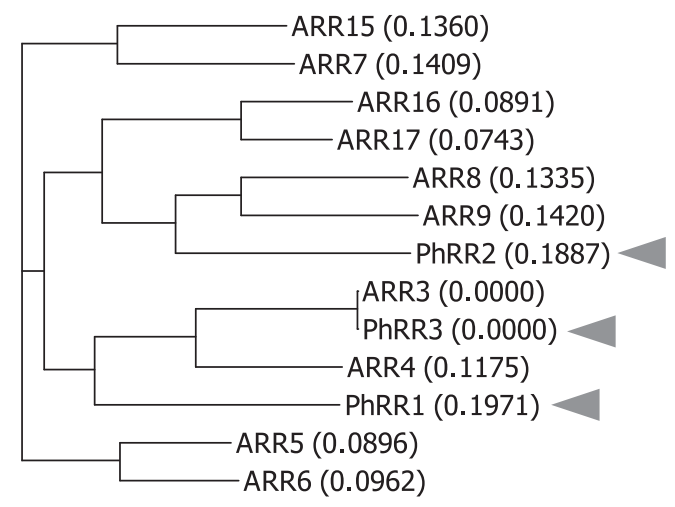

B

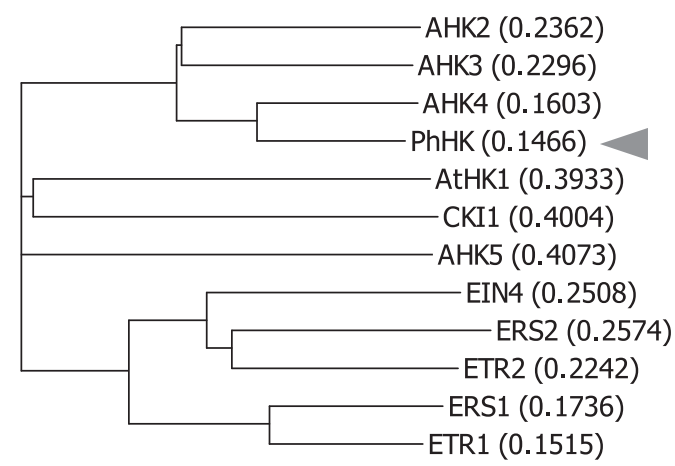

Fig. 1. Phylogenic relationships of type-A response regulator (A) and histidine protein kinase (B) in petunia and Arabidopsis. Mediumflowered 'Baccara White' and large-flowered 'Fulcon Midblue' (Table 1) were used for isolation of petunia full-length cDNA. PhRRs, petunia type-A response regulators; ARRs, Arabidopsis type-A response regulators (ARR3, Atlg59940; ARR4, At1g104700; ARR5, At3g48100; ARR6, At5g62920; ARR7, At1g19050; ARR8, At2g41310; ARR9, At3g57040; ARR15, Atlg74890; ARR16, At2g40670; ARR17, At3g56380); PhHK, petunia cytokinin receptor histidine protein kinase; AHK2-4 (At5g35750, Atlg27320, At2g01830, respectively), Arabidopsis cytokinin receptor histidine protein kinases. AtHK1 (At2g17820), CKI1 (At2g47430), AHK5 (At5g10720), EIN4 (At3g04580), ERS1/2 (At2g40940, At1g04310, respectively), and ETR1/2 (At1g66340, At3g23150, respectively) are Arabidopsis histidine protein kinases with functions other than cytokinin receptors. Gray arrows indicate petunia proteins. Values in paretheses indicate P-distance. domains sequentially from the N-terminal: hydrophobic membrane-spanning domain, CHASE domain, hydrophobic membrane-spanning domain, histidine kinase domain, receiver-like domain, and receiver domain (Ueguchi et al., 2001). The phylogenic relationship among PhHK, Arabidopsis CK receptor HKs, and other HKs is shown in Figure 1B. PhHK belonged to the same clade as Arabidopsis CK receptor HKs (i.e., AHK2-4), and was different from HKs with other functions. PhHK had the closest relationship with AHK4 among the three Arabidopsis CK receptor HKs. Deduced amino acid sequences unique to large-flowered cultivars were not detected in PhRR1-3 and PhHK.

Expression of PhRR 1-3 was upregulated within $1 \mathrm{~h}$ of BA application and then gradually decreased (Fig. 2). Expression of $\mathrm{PhHK}$ was also upregulated within $1 \mathrm{~h}$ of BA application, and the upregulation continued for at least $24 \mathrm{~h}$ after BA application. These rapid upregulations of type-A RRs and CK receptor $H K$ are common in Arabidopsis type-A RRs (Brandsttatter and Kieber, 1998; Taniguchi et al., 1998) and a CK receptor $H K, A H K 4$ (Kiba et al., 2004; Rashotte et al., 2003). The structural similarity to the corresponding proteins in Arabidopsis and the rapid response to CK suggest that $P h R R 1-3$ and $P h H K$ function as type-A $R R s$ and $\mathrm{CK}$ receptor $H K$, respectively.

\section{Expression analysis of type- $A R R$ and $C K$ receptor $H K$} genes among cultivars and developmental stages

Expressions of PhRR 1-3 and $P h H K$ were not markedly different between small- and medium-flowered $g g$ cultivars throughout corolla development (Fig. 3); however, expression levels of PhRRl and PhRR2 were conspicuously higher in large-flowered $G g$ cultivars than in small- and medium-flowered $g g$ cultivars. These high

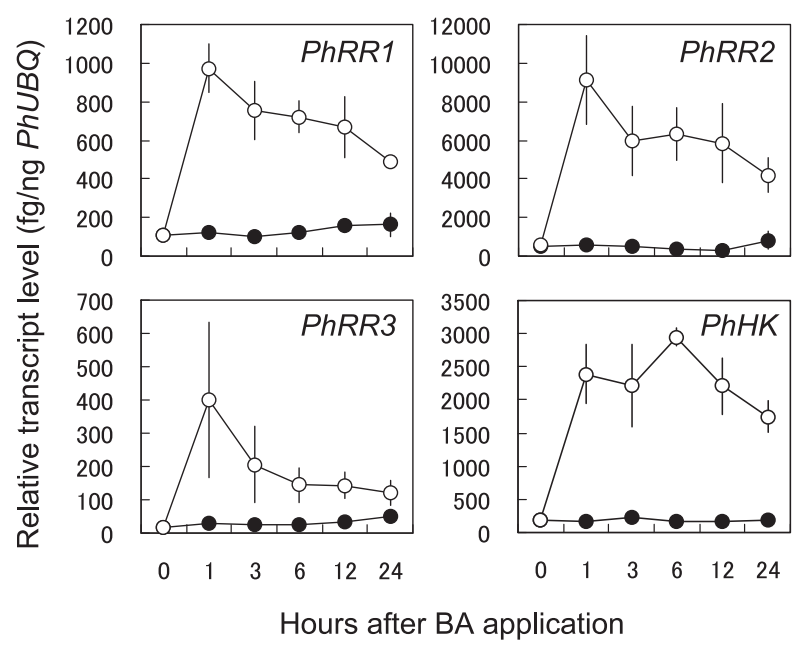

Fig. 2. Effect of application of $N^{6}$-benzylaminopurine (BA) on the expression of genes encoding type-A response regulator and cytokinin receptor histidine protein kinase. PhRRs, petunia typeA response regulator genes; $P h H K$, petunia cytokinin receptor histidine protein kinase gene. Symbols indicate control $(\mathbf{O})$ and BA-treated $(\bigcirc)$ corollas. Vertical bars represent $\pm \operatorname{SE}(n=3)$. 


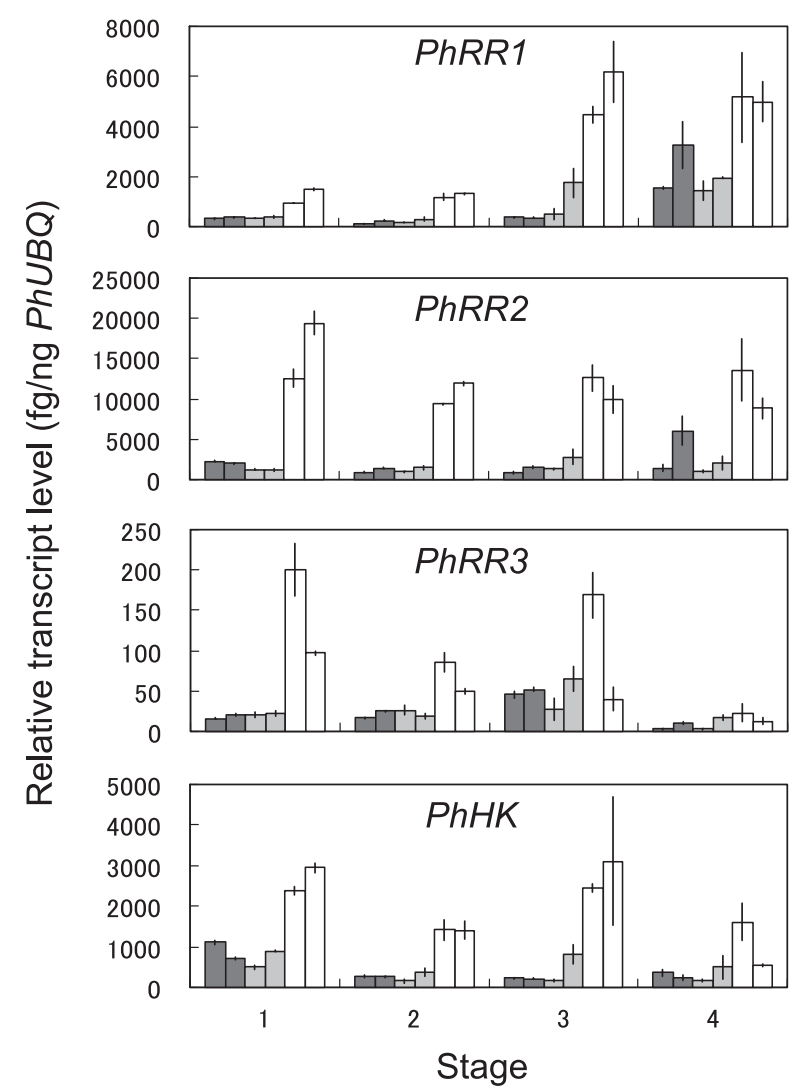

Fig. 3. Expression of genes encoding type-A response regulator and cytokinin receptor histidine protein kinase during corolla development in small-, medium-, and large-flowered cultivars. $P h R R s$, petunia type-A response regulator genes; $P h H K$, petunia cytokinin receptor histidine protein kinase gene. Developmental stage of corolla: stage 1 , early cell proliferation stage; stage 2 , late cell proliferation stage; stage 3 , cell expansion stage; stage 4, flower opening. Dark gray column, small-flowered cultivars (left, 'Fantasy Ivory'; right, 'Fantasy Blue'); light gray column, medium-flowered cultivars (left, 'Baccara White'; right, 'Baccara Blue Picotee'); open column, large-flowered cultivars (left, 'Fulcon White'; right, 'Fulcon Midblue'). Vertical bars represent $\pm \operatorname{SE}(n=3)$.

expression levels, unique to large-flowered $G g$ cultivars, were marked at stages 1 and 2, and persisted until stage 3 , while they were not obvious at stage 4. Expression level of PhRR3 was also higher in large-flowered $G g$ cultivars than in small- and medium-flowered $g g$ cultivars in stages 1 and 2, while this high expression level was not obvious in stages 3 and 4. Large-flowered $G g$ cultivars also exhibited a higher expression level of $P h H K$ than small- and medium-flowered $g g$ cultivars in stages $1-3$, while it was not obvious in stage 4 . Therefore, $G g$-cultivar-specific high expression of type-A $R R s$ and $\mathrm{CK}$ receptor $H K$ was conspicuous in the cell proliferation stages. This high expression continued until the cell expansion stage, although there was an exception (i.e., expression of PhRR3 in this stage).

To examine whether the high expression levels of these genes is a common phenomenon among largeflowered $G g$ cultivars, we also analyzed the corollas of

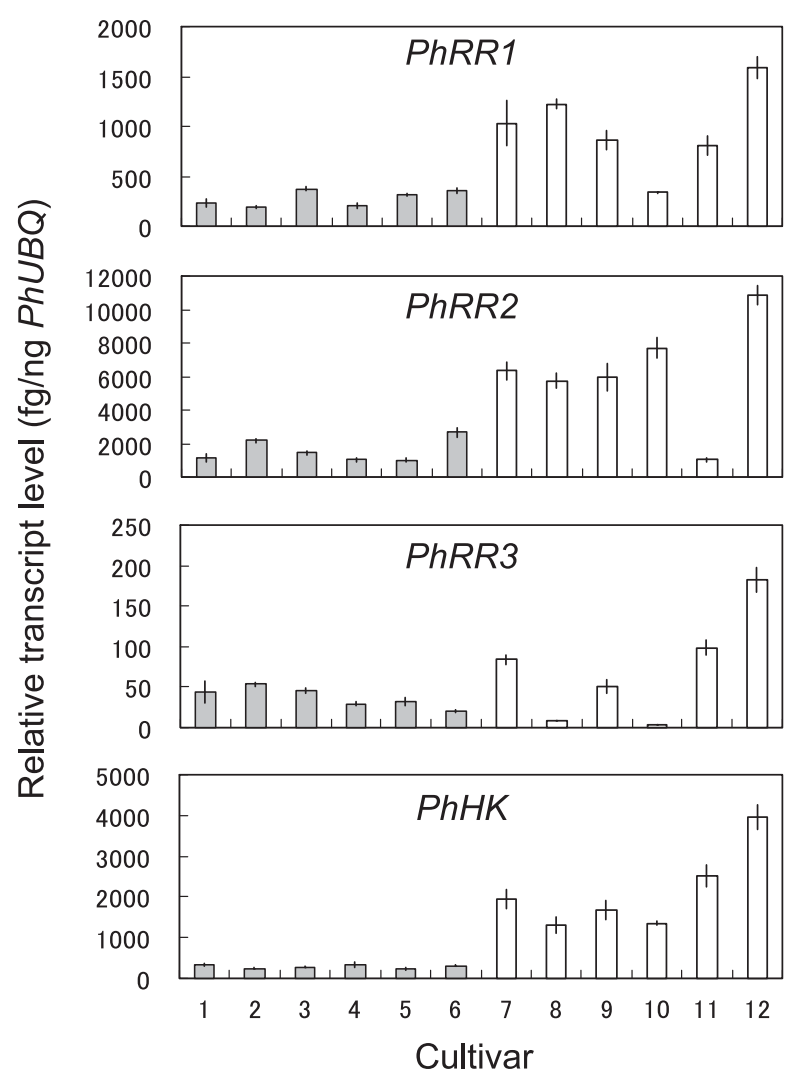

Fig. 4. Expression of genes encoding type-A response regulator and cytokinin receptor histidine protein kinase in corollas of various medium- and large-flowered cultivars. Corollas at stage 2, late cell proliferation stage, were used. $P h R R s$, petunia type-A response regulator genes; $P h H K$, petunia cytokinin receptor histidine protein kinase gene. Gray column, medium-flowered cultivars (1, 'Pearl White'; 2, 'Pearl Sky Blue'; 3, 'Baccara Rose Morn'; 4, 'Carpet Pink'; 5, 'Rondo Pink'; 6, 'Rondo Violet Imp'); open column, large-flowered cultivars (7, 'Fulcon Pink Morn'; 8, 'Carnival Red Star'; 9, 'Carnival Brush Pink'; 10 , 'Supercascade Blue'; 11, 'Carnival Salmon'; 12, 'Dream White'). Vertical bars represent $\pm \mathrm{SE}(\mathrm{n}=3)$.

12 other cultivars at stage 2 (Fig. 4). In several largeflowered $G g$ cultivars, some PhRRs did not show marked higher expression than in medium-flowered $g g$ cultivars (i.e., $P h R R 1$ and $P h R R 3$ in 'Supercascade Blue', PhRR2 in 'Carnival Salmon', and $P h R R 3$ in 'Carnival Red Star'); however, at least one of PhRR1-3 had markedly higher expression levels in each of the large-flowered $G g$ cultivars than in the medium-flowered $g g$ cultivars. In Arabidopsis, 10 type-A $R R \mathrm{~s}$ function in partial redundancy as negative regulators of $\mathrm{CK}$ signaling (Imamura et al., 1999; To et al., 2004), suggesting that $P h R R 1-3$ also functions redundantly. Even if the expression of a certain type-A $R R$ is inhibited by mutation, other type-A $R R$ s will compensate for the function, as shown by To et al. (2004). Thus, high expression of typeA $R R \mathrm{~s}$ is probably a common phenomenon among large-flowered $G g$ cultivars; nevertheless, some type-A $R R s$ have lost the response to the large-flowered genotype, possibly through mutation. On the other hand, expression of $\mathrm{PhHK}$ was consistently higher in large- 


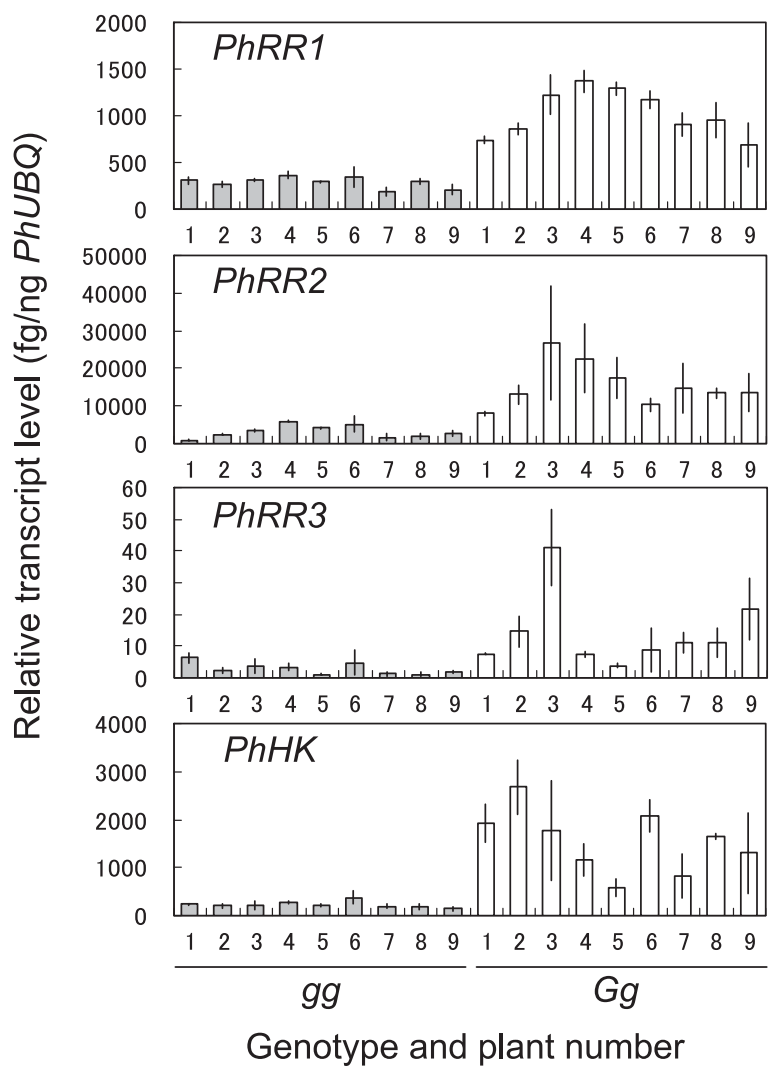

Fig. 5. Expression of genes encoding type-A response regulator and cytokinin receptor histidine protein kinase in corolla of 'Mitchell' $\mathrm{BC}_{4}$ plants with $g g$ (gray column) and $G g$ (open column) genotypes. Corollas at stage 2, late cell proliferation stage, were used. PhRRs, petunia type-A response regulator genes; $P h H K$, petunia cytokinin receptor histidine protein kinase gene. Vertical bars represent $\pm \operatorname{SE}(n=3)$.

flowered $G g$ cultivars than in medium-flowered $g g$ cultivars, suggesting that high expression of $\mathrm{PhHK}$ is also a common phenomenon among large-flowered $G g$ cultivars.

To confirm that high expressions of PhRRs and PhHK are caused by the $G g$ genotype, we performed expression analysis employing $\mathrm{BC}_{4}$ plants in which the genetic backgrounds except for the $G$ allele were mostly as same as that of a doubled haploid line 'Mitchell'. All of the $9 \mathrm{BC}_{4}$ plants with $G g$ genotype exhibited higher expression levels of PhRR1,PhRR2, and PhHK than the 9 plants with $g g$ genotype (Fig. 5). The expression level of $P h R R 3$, which was much lower than PhRRl and $P h R R 2$, was also conspicuously higher in most $G g$ plants than in $g g$ plants, although some $G g$ plants had about the same expression levels as those of $g g$ plants. These results strongly suggest that high expression levels of type-A RRs and CK receptor $H K$ are induced by the $G$ allele.

Expression levels of $P h R R 1-3$ and $P h H K$ in each organ were compared between $\mathrm{BC}_{4}$ plants with $G g$ and $g g$ genotypes. Higher expression levels of PhRRI-3 and $P h H K$ in $G g$ than in $g g$ plants were observed in the sepals and stamens (Fig. 6). These genes, except for

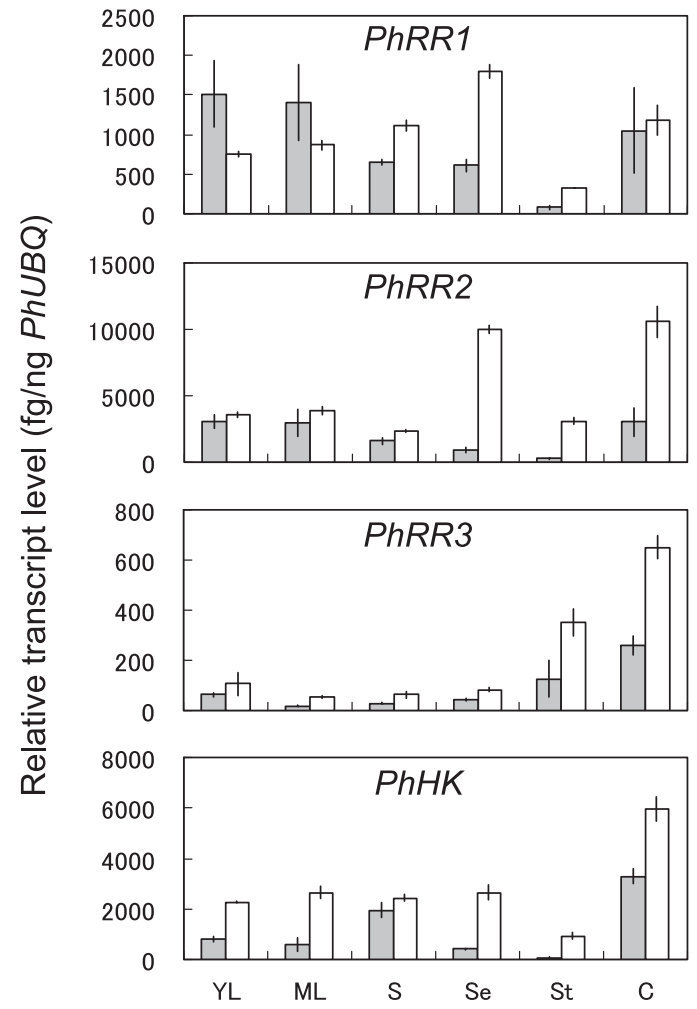

Fig. 6. Expression of genes encoding type-A response regulator and cytokinin receptor histidine protein kinase in various organs of 'Mitchell' $\mathrm{BC}_{4}$ plants with $g g$ (gray column) and $G g$ (open column) genotypes. PhRRs, petunia type-A response regulator genes; $P h H K$, petunia cytokinin receptor histidine protein kinase gene. YL, young leaf; ML, mature leaf; S, stem; Se, sepal; St, stamen; C, carpel. The floral organs were collected form the flower bud in stage 2. Vertical bars represent $\pm \operatorname{SE}(n=3)$.

$P h R R 1$, were also promoted by the $G g$ genotype in the carpels. In contrast, ambiguous results were obtained for their expression in leaves and stems. In leaves, the expression of $P h R R l$ was higher in $g g$ than in $G g$ plants, although the $G g$ genotype promoted $P h H K$ expression. In stems, the expression levels of PhRRI-3 and PhHK were not markedly different between $g g$ and $G g$ plants. Thus, the $G$-allele-induced high expression of type-A $R R$ and CK receptor $H K$ was consistent in floral organs, but to some extent, it was more ambiguous in vegetative organs.

Accumulation of type-A RR serves as a negative regulator of CK signaling in the phosphorelay circuitry (Rashotte et al., 2003). In contrast, the effects of a high expression of $\mathrm{CK}$ receptor $H K$ have not been well documented. HK is activated by phosphorylation of the receiver domain by the HK domain (Stock et al., 2000). The HK and receiver domains also have phosphatase activity that dephosphorylate the receiver domain and inactivate HK (Stock et al., 2000). Owing to these contradictory actions, the possibility that a high expression level of PhHK induces the enhanced CK response and enlarge corolla can not be ruled out; therefore, we constructed transgenic plants overexpress- 
ing $\mathrm{PhHK}$ under the control of CaMV $35 \mathrm{~S}$ promoter. However, overexpression of $\mathrm{PhHK}$ did not induce the enlargement of any organs, including the corolla (Nishijima et al., unpublished data), suggesting that a high expression level of PhHK is not the cause of the large-flowered phenotype induced by the $G$ allele.

Role of the G-allele-induced promotion of type- $A R R$ and $C K$ receptor $H K$ genes in corolla enlargement

The effects of CK application and the $G$ allele on the CK phosphorelay signaling circuitry and corolla enlargement are summarized in Figure 7 . The $G$-alleleinduced high expression of type-A $R R s$ in the petunia corolla probably represses the $\mathrm{CK}$ response in $\mathrm{CK}$ signaling as described above, although the function of the high expression of $P h H K$ remains unclear. The $G$ allele also decreased endogenous CK concentration in the corolla through enhanced expression of CKX genes (Nishijima et al., 2011). These changes in CK biosynthesis and the phosphorelay signaling circuitry resemble the changes induced by the CK response (Kiba et al., 2005). Thus, it can be assumed that the $G$ gene has a mutation that promotes the CK response (Fig. 7, arrow 1). Although the mutation might be present in the CK phosphorelay signaling circuitry, $P h H K$ may not be the causal gene because overexpression of $P h H K$ had no effect on corolla enlargement, as mentioned above. It is also possible that the mutation is present in the factor promoting the $\mathrm{CK}$ response in $\mathrm{CK}$ signaling in trans, as shown in the overexpression of Arabidopsis CKI1 (Kakimoto, 1996).

On the other hand, the concept that the $G$ allele enlarges the corolla independently of the CK phospho-

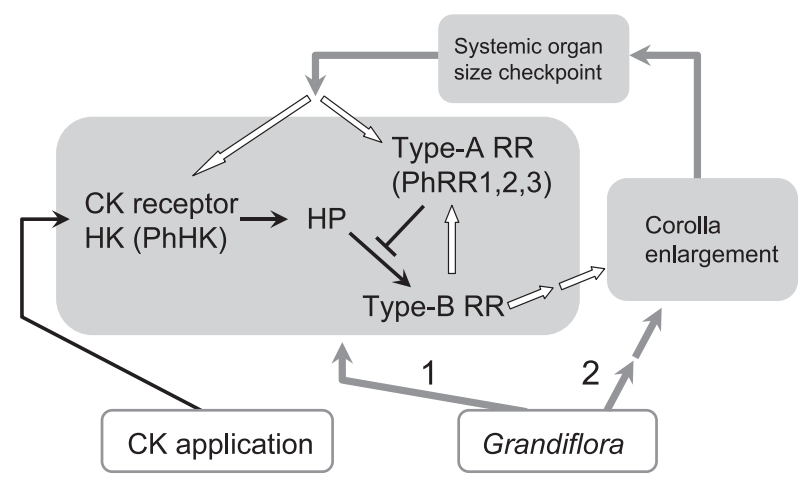

Fig. 7. Cytokinin (CK) phosphorelay signaling circuitry regulated by Grandiflora and cytokinin application in petunia corolla. HK, histidine protein kinase; PhHK, petunia CK receptor HK; HP, histidine phosphotransfer protein; $\mathrm{RR}$, response regulator; $\mathrm{PhRR}$, petunia type-A RR. Black arrows indicate promotion $(\rightarrow)$ and inhibition $(-)$ ) of signaling mediated by direct molecular interaction. Open arrows indicate promotion of gene expression. Gray arrows indicated by 1 and 2 show hypothetical pathways regulating CK phosphorelay signaling circuitry by Grandiflora. Note that target of the pathway 1 is not either cirtain protein or gene but CK phosphorelay signaling circuitry. The illustration of the CK signaling phosphorelay circuitry is based on Oka (2005) and Mizuno (2005). relay signaling circuitry cannot be excluded (Fig. 7, arrow 2). This is because the repression of CK signaling possibly occurs as negative feedback from the hypothesized systemic checkpoint of the organ size that senses excess organ enlargement (Weiss et al., 2005). In either case, it is clear that the $G$ allele causes feedback regulation of the $\mathrm{CK}$ phosphorelay circuitry to partially repress $\mathrm{CK}$ signaling through promotion of type-A RRs.

The $G$ allele induces large and thick vegetative organs (i.e., leaf and stem) as well as large and thick floral organs (i.e., sepal and corolla) (Ewart, 1984), however, the $G$-allele-induced high expression levels of PhRRs and $P h H K$ were more consistent in floral organs than in vegetative organs (Fig. 5). This result may have different implications depending on the assumptions described above. Based on the former assumption that the $G$ allele promotes a CK response, the floral organ-preferable high expression of PhRRs and PhHK might reflect the greater $C K$ response in floral organs than in vegetative organs. This presumption corresponds with the observation that $G$ allele enlarges floral organs like sepals and corolla to a greater extent than leaves.

In contrast, based on the latter assumption that $G$ allele induces organ enlargement independently of CK phosphorelay signaling circuitry and high expression of PhRRs and PhHK is caused by feedback regulation from the systemic organ size checkpoint, the floral organpreferable high expression of PhRRs and PhHK might reflect strict feedback regulation of floral organ size. In wild petunia species, including P.axillaries and P. integrifolia, which are ancestors of P. hybrida, the flower size and shape are critical evolutionary traits for adaptation to specific pollinators (Ando et al., 2001; Galliot and Hoballah, 2006); therefore, "incorrect" flower size causes severe disadvantage in propagation. This evolutionary background might have conferred the strict regulation of flower size.

\section{Potential modification of CK phosphorelay signaling in petunia breeding}

The $G$ gene is the only major gene inducing largeflowered phenotype in petunia (Ewart, 1984). Although this gene effectively induces a large-flowered phenotype, it has several pleiotropic effects, as described above. To establish larger-flowered phenotypes of petunia, it is necessary to find another large-flower mutation or a modification mutant that enhances the $G$-allele-induced large-flowered phenotype. As a modification mutation, the repression of $G$-allele-induced feedback regulation of CK biosynthesis and phosphorelay signaling may be effective, because this feedback regulation represses the $\mathrm{CK}$ response, which induces the large-flowered phenotype (Nishijima et al., 2006). A possible way to repress the feedback regulation of CK biosynthesis was described previously (Nishijima et al., 2011). To repress the feedback regulation of the $\mathrm{CK}$ phosphorelay signaling circuitry, the presence of type-A $R R s$ that have 
lost their response to the $G$ allele (Fig. 4) will be key tools. In Arabidopsis, a reduction in type-A RR function enhanced the CK response (To et al., 2004). Since there are 10 type-A RRs in Arabidopsis with partial functional redundancy (Imamura et al., 1999), accumulation of multiple mutant genes was necessary to enhance the $\mathrm{CK}$ response (To et al., 2004). Type-A RRs, other than $P h R R 1-3$, are likely to be present in petunia. Analysis of all of the type-A $R R s$ expressed in the corolla may enable us to find and accumulate type-A $R R s$ which are unresponsive to the $G$ allele. Large-flowered cultivars actually have the potential for further corolla enlargement through increased $\mathrm{CK}$ response, because $\mathrm{CK}$ application further enlarges the corolla of large-flowered cultivars with $G g$ genotype (Nishijima et al., 2006).

\section{Acknowledgements}

We thank Mrs. T. Kurobe for the technical assistance.

\section{Literature Cited}

Ando, T., M. Nomura, J. Tsukahara, H. Watanabe, H. Kokubun, T. Tsukamoto, G. Hashimoto, E. Marchesi and J. Kitching. 2001. Reproductive isolation in a native population of Petunia sensu Jussieu (Solanaceae). Ann. Bot. 88: 403-413.

Brandstatter, I. and J. J. Kieber. 1998. Two genes with similarity to bacterial response regulators are rapidly and specifically induced by cytokinin in Arabidopsis. Plant Cell 10: 10091019.

Ewart, L. 1984. Plant Breeding. p. 180-253. In: K. C. Sink (ed.). Petunia. Springer-Verlag, Berlin.

Frohman, M. A., M. K. Dush and G. R. Martin. 1988. Rapid production of full-length cDNAs from rare transcripts: amplification using a single gene-specific oligonucleotide primer. Proc. Natl. Acad. Sci. USA 85: 8998-9002.

Galliot, C. and M. E. Hoballah. 2006. Genetics of flower size and nector volume in Petunia pollination syndrome. Planta 225: 203-212.

Imamura, A., N. Hanaki, A. Nakamura, T. Suzuki, M. Taniguchi, T. Kiba, C. Uesugi, T. Sugiyama and T. Mizuno. 1999. Compilation and characterization of Arabidopsis thaliana response regulators implicated in His-Asp phosphorelay signal transduction. Plant Cell Physiol. 40: 733-742.

Kakimoto, T. 1996. CKI1, a histidine kinase homolog implicated in cytokinin signal transduction. Science 274: 982-985.

Kiba, T., K. Aoki, H. Sakakibara and T. Mizuno. 2004. Araidopsis response regulator, ARR22, ectopic expression of which results in phenotypes similar to the wol cytokinin-receptor mutant. Plant Cell Physiol. 45: 1063-1077.

Kiba, T., T. Naitou, N. Koizumi, T. Yamashino, H. Sakakibara and T. Mizuno. 2005. Combinatorial microarray analysis revealing Arabidopsis genes implicates in cytokinin responses through the His $\rightarrow$ Asp phosphorelay circuitry. Plant Cell Physiol. 46: 339-355.

Leibfrield, A., J. P. C. To, W. Busch, A. Stehling, A. Kehle, M.
Demar, J. J. Kieber and U. Lohmann. 2005. WUSCHEL control meristem function by direct regulation of cytokinininducible response regulators. Nature 438: 1172-1175.

Mizuno, T. 2005. Two-component phosphorelay signal transduction systems in plants: From hormone responses to circadian rhythms. Biosci. Biochem. Biotech. 69: 2263-2276.

Nishijima, T. 2007. Hanagata. p. 37-43. In: Nosangyosonbunkakyokai (ed.). Nogyogijutsutaikei. Kakihen (Tsuiroku). 9 (In Japanese). Nosangyosonbunkakyokai, Tokyo.

Nishijima, T., H. Miyaki, K. Sasaki and T. Okazawa. 2006. Cultivar and anatomical analysis of corolla enlargement of petunia (Petunia hybrida Vilm.) by cytokinin application. Sci. Hortic. 111: 49-55.

Nishijima, T., T. Niki and T. Niki. 2011. Corolla of the largeflowered petunia (Petunia hybrida Vilm.) cultivars exhibit low endogenous cytokinin concentration through enhanced expression of the genes encoding cytokinin oxidases. J. Japan. Soc. Hort. Sci. 80: 334-342.

Oka, A. 2005. Cytokinin signal transduction and two-component regulatory system. Gamma Field Symp. 42: 25-39.

Rashotte, A. M., S. D. B. Carson, J. P. C. To and J. J. Kieber. 2003. Expression profiling of cytokinin action in Arabidopsis. Plant Physiol. 132: 1998-2011.

Saitou, N. and M. Nei. 1987. The neighbor-joining method: a new method for reconstructing guide trees. Mol. Biol. Evol. 4: 406-425.

Stock, A. M., V. L. Robinson and P. N. Goudreau. 2000. Twocomponent signal transduction. Annu. Rev. Biochem. 69: 183-215.

Sweere, U., K. Eichenberg, J. Lohrmann, V. Mira-Ronado, I. Bäurle, J. Kudla, F. Nagy, E. Schäfer and K. Harter. 2001. Interaction of the response regulator ARR4 with phytochrome B in modulating red light signaling. Science 294: 1108-1111.

Taniguchi, M., T. Kiba, H. Sakakibara, C. Ueguchi, T. Mizuno and T. Sugiyama. 1998. Expression of Arabidopsis response regulator homologs is induced by cytokinins and nitrate. FEBS Lett. 429: 259-262.

To, J. P. C., G. Haberer, F. J. Ferreira, J. Deruere, M. G. Mason, G. E. Schaller, J. M. Alonso, J. R. Ecker and J. J. Kieber. 2004. Type-A Arabidopsis response regulators are partially redundant negative regulators of cytokinin signaling. Plant Cell 16: 658-671.

Ueguchi, C., H. Koizumi, T. Suzuki and T. Mizuno. 2001. Novel family of sensor histidine kinase genes in Arabidopsis thaliana. Plant Cell Physiol. 42: 231-235.

Verdonk, J. C., K. Shibuya, H. M. Loucas, T. A. Colquhoun, A. Underwood and D. G. Clark. 2008. Flower-specific expression of the Agrobacterium tumefaciens isopentenyltransferase gene results in radial expansion of floral organs in Petunia hybrida. Plant Biotech. J. 6: 694-701.

Weiss, J., L. Delgado-Benerroch and M. Egea-Cortines. 2005. Genetic control of floral size and proportions. Int. J. Dev. Biol. 49: 513-525.

Zhao, Z., S. U. Andersen, K. Ljung, K. Dolezal, A. Miotk, S. J. Schultheiss and J. U. Lohmann. 2010. Hormonal control of the shoot stem-cell niche. Nature 465: 1089-1093. 